\title{
Regulation of Crossover Frequency and Distribution during Meiotic Recombination
}

\author{
Takamune T. Saito and Monica P. Colaiácovo \\ Department of Genetics, Harvard Medical School, Boston, Massachusetts 02115 \\ Correspondence: mcolaiacovo@genetics.med.harvard.edu
}

\begin{abstract}
Crossover recombination is essential for generating genetic diversity and promoting accurate chromosome segregation during meiosis. The process of crossover recombination is tightly regulated and is initiated by the formation of programmed meiotic DNA double-strand breaks (DSBs). The number of DSBs is around 10-fold higher than the number of crossovers in most species, because only a limited number of DSBs are repaired as crossovers during meiosis. Moreover, crossovers are not randomly distributed. Most crossovers are located on chromosomal arm regions and both centromeres and telomeres are usually devoid of crossovers. Either loss or mislocalization of crossovers frequently results in chromosome nondisjunction and subsequent aneuploidy, leading to infertility, miscarriages, and birth defects such as Down syndrome. Here, we will review aspects of crossover regulation observed in most species and then focus on crossover regulation in the nematode Caenorhabditis elegans in which both the frequency and distribution of crossovers are tightly controlled. In this system, only a single crossover is formed, usually at an off-centered position, between each pair of homologous chromosomes. We have identified C. elegans mutants with deregulated crossover distribution, and we are analyzing crossover control by using an inducible single DSB system with which a single crossover can be produced at specific genomic positions. These combined studies are revealing novel insights into how crossover position is linked to accurate chromosome segregation.
\end{abstract}

Meiosis is a specialized cell division process that generates haploid gametes from diploid parental germ cells. This reduction in the number of chromosomes is achieved by following a single round of DNA replication with two consecutive cell divisions (meiosis I and II). Homologous chromosomes are separated at meiosis I, and sister chromatids are separated at meiosis II. There are unique chromosomal events that need to take place during prophase to ensure that homologs segregate properly at meiosis I (Fig. 1). Homologous chromosomes need to find each other and pair; these pairing interactions need to be stabilized via the formation of a scaffold known as the synaptonemal complex, which assembles at the interface between paired homologs; and interhomolog recombination needs to take place in order to produce crossovers. Crossover formation is one of the sources of genetic diversity in the population. Moreover, crossovers result in physical attachments (chiasmata) between homologs that, underpinned by cohesion, confer the tension required to properly align the attached homologs (bivalents) at the metaphase plate and then orient them toward opposite poles of the meiosis I spindle.

Errors in crossover formation result in chromosome nondisjunction leading to aneuploidy, which causes infertility, miscarriages, birth defects, and cancers.

Given the impact of crossover formation on human health and reproductive biology, it is therefore not surprising that crossovers are tightly regulated. For example, crossover formation is not frequently observed near centromeres and telomeres, suggesting they may be repressed in these regions. Crossovers at centromere regions lead to aneuploidy in female meiosis and crossovers at telomeres increase azoospermia (Ottolini et al. 2015; Ren et al. 2016). However, direct testing of how a crossover positioned near centromeres or telomeres might lead to increased errors in chromosome segregation has been challenging in metazoans.

Caenorhabditis elegans is an ideal model organism to study crossover control, because crossover formation is tightly regulated in comparison to other known model organisms. A single off-centered crossover is formed on each of the six pairs of homologous chromosomes in C. elegans compared to the one to four crossovers per pair of homologs observed in other species (Barnes et al. 1995; Martinez-Perez and Colaiácovo 2009; Rockman and Kruglyak 2009). Surprisingly, a single DNA double-strand break (DSB) is sufficient to make a crossover in C. elegans (Rosu et al. 2011). This property, coupled with the use of a system in which a single DSB can be induced at defined genomic positions, allows us to analyze how crossover position affects meiotic chromosome segregation in C. elegans. Here, we review what is known for crossover control from studies in different organisms, our novel findings regarding regulation of crossover position using the single inducible DSB system in C. elegans, and the future directions of research using this system aimed at understanding the origin of aneuploidies.

\section{MOLECULAR STEPS IN CROSSOVER FORMATION}

Crossover formation starts with the formation of DSBs by a topoisomerase-like protein present from yeast to hu-

(C) 2017 Saito and Colaiácovo. This article is distributed under the terms of the Creative Commons Attribution-NonCommercial License, which permits reuse and redistribution, except for commercial purposes, provided that the original author and source are credited. 


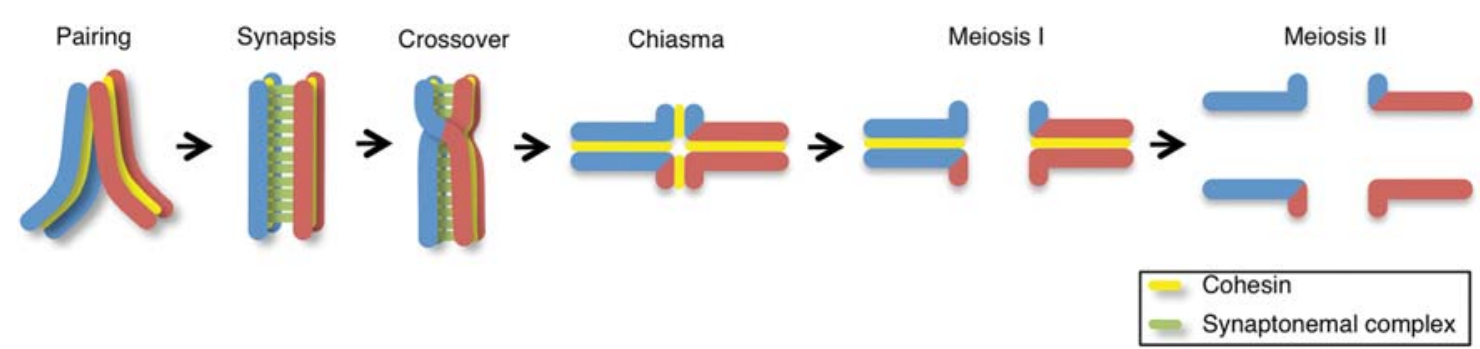

Figure 1. Meiosis and crossover formation. Chromosome dynamics during meiosis. After premeiotic DNA replication, homologous chromosomes find each other (pairing) during the leptotene-zygotene stages. The synaptonemal complex assembles, aligning and holding homologs together throughout their full lengths (synapsis) at the pachytene stage. Repair of DNA double-strand breaks (DSBs) via crossover formation involves the reciprocal exchange of genetic information between homologs. A chiasma is the cytologically visible manifestation of an earlier crossover event underpinned by flanking sister chromatid cohesion and is observed as a cruciform configuration during the diplotene to diakinesis transition. Homologous chromosomes are segregated at the metaphase I to anaphase I transition, and sister chromatids are separated at the metaphase II to anaphase II transition. Paternal chromatids are blue, and maternal chromatids are red. Sister chromatid cohesion is depicted in yellow, and the synaptonemal complex is depicted in green.

mans known as Spo11 (Fig. 2; Keeney et al. 1997). DSBs then undergo $5^{\prime}$-end resection to produce $3^{\prime}$ overhangs through the activity of the Mre11/Rad50/Xrs2 exonuclease complex. Rad51 associates with the $3^{\prime}$ single-stranded DNA overhangs producing a DNA-protein filament that then engages in a search for homologous DNA sequences. The $3^{\prime}$ end invades the homologous template (singlestrand invasion resulting in D-loop formation), followed by DNA synthesis. At this point, repair can proceed through different pathways resulting in either the displacement and annealing of the newly synthesized strand to its complementary strand (synthesis-dependent strand annealing [SDSA]) (Fig. 2A) or in second end capture to produce a double Holliday junction (dHJ) intermediate (Fig. 2B). The SDSA pathway results only in noncrossover products. Meanwhile, the asymmetric resolution of dHJs by structure-specific endonucleases (Slx1-Slx4, Mus81-Mms4, and Yen1) produces crossover products (Fig. 2C), whereas their symmetric resolution results in noncrossovers (Fig. 2D). It has been proposed that designated and nondesignated DSBs are converted to dHJs and resolved by different structure-specific endonucleases in yeast to assure and limit the number of crossovers (Zakharyevich et al. 2012). dHJs can also be processed through dissolution mediated by the Sgs1-Top3-Rmi1 complex during which the two Holliday junctions branch migrate toward one another until they form a hemicatenated intermediate that can be decatenated by topoisomerase III, resulting in noncrossovers (Fig. 2E). Finally, all DSBs undergoing intersister repair result in noncrossovers. This detailed blueprint of the molecular requirements for crossover formation allows for assessment of how these may differ during DSB repair depending on the location of the DSB in a metazoan.

\section{CLASS I AND CLASS II CROSSOVERS}

Two different classes of crossovers have been identified, namely class I and class II. In yeast, around 90 crossovers are observed in 16 bivalents. Seventy percent (range from $60 \%$ to $90 \%$ ) of crossovers are class I crossovers, which are dependent on the meiosis-specific ZMM pro- teins (Zip1, Zip2, Zip3, Msh4, Msh5, Mer3, Spo16, and Spo22/Zip4). Zip1 is a structural component of the synaptonemal complex that holds pairs of homologous chromosomes together. Zip2 is a XPF-like helix-hairpin-helix containing protein (Chua and Roeder 1998; Macaisne et al. 2008) and Zip3 is a SUMO E3 ligase, whereas Msh4 and Msh5 are homologs of the Escherichia coli mismatch repair protein MutS implicated in stabilizing dHJ intermediates (Snowden et al. 2004). The number of class I crossovers (approximately 60) matches the number of Zip2, Zip3, and Msh4-Msh5 foci observed in yeast pachytene nuclei. Mer3 is a DNA helicase required for Holliday junction branch migration (Nakagawa and Ogawa 1999; Mazina et al. 2004). Finally, Spo16 and Spo22/Zip4 are involved in synaptonemal complex assembly, and they are unique because, in contrast to the other ZMM proteins, they are not essential for crossover interference (Shinohara et al. 2008) (see the subsection Crossover Interference). The remaining $\sim 30 \%$ of crossovers fall into class II. Class II crossovers depend on double Holliday junction resolution executed by the structure-specific endonucleases Mus81-Mms4, Slx1-Slx4, and Yen1 in yeast (Fig. 2C,D; Zakharyevich et al. 2012).

\section{MULTIPLE LAYERS OF CROSSOVER REGULATION}

\section{Crossover Assurance/Obligate Crossover}

Because crossover formation is essential for proper chromosome segregation at meiosis I (Fig. 1), at least one crossover (obligate crossover) has to be formed between each pair of homologous chromosomes. This phenomenon is called "crossover assurance" (Fig. 3A).

A notable exception to this regulation is observed in Drosophila in which both male meiosis and female chromosome 4 are devoid of crossover formation (Cooper 1949; Hartmann and Sekelsky 2017) and chromosome segregation occurs randomly at meiosis II. In contrast, recombinant chromatids are preferentially segregated to oocytes and not into the second polar body in human female meiosis (Ottolini et al. 2015). Robust crossover assurance is also observed in C. elegans, where only 


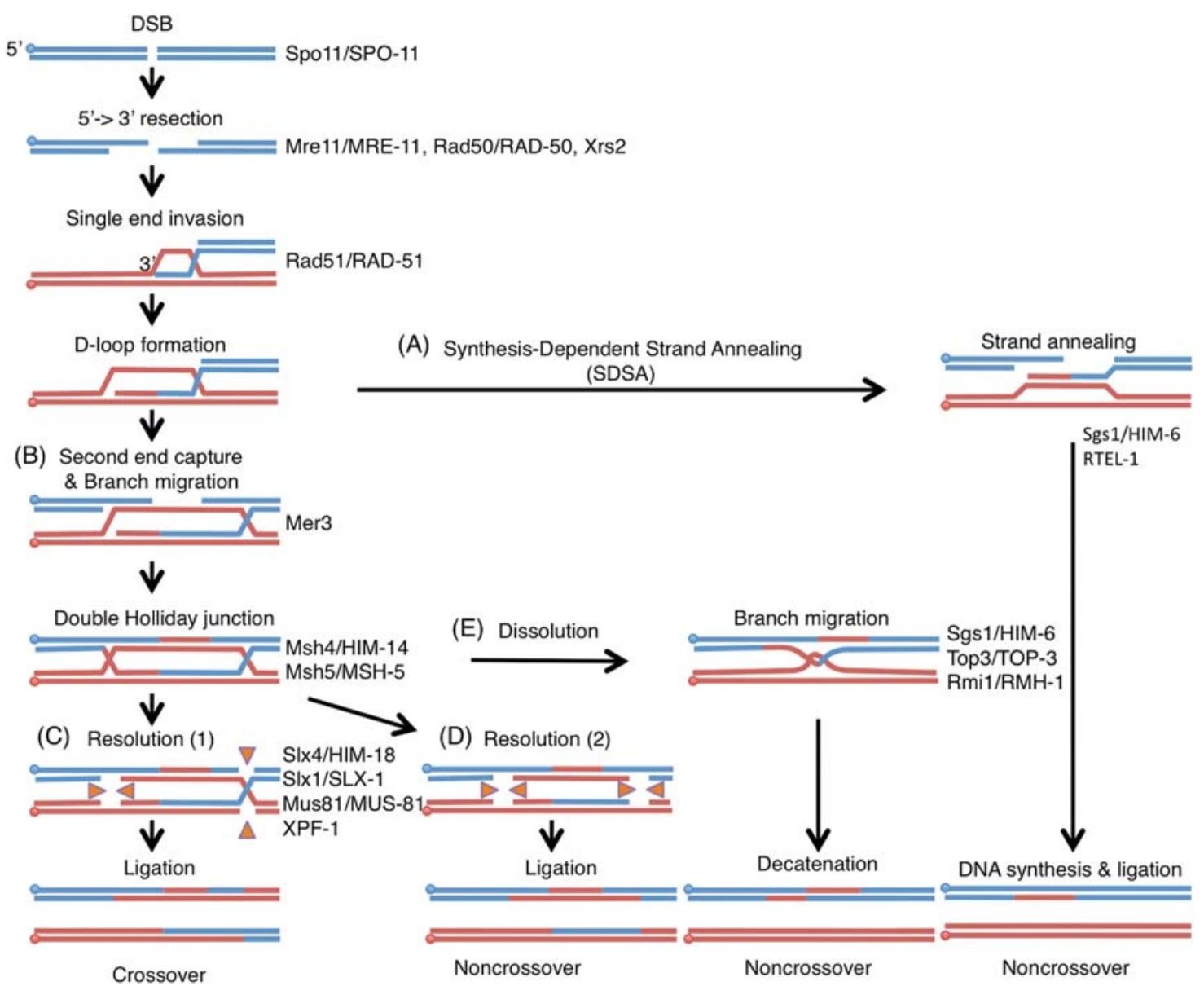

Figure 2. Model of homologous recombination. DNA double strand breaks are generated by the topoisomerase-like protein Spo11. The MRN/X complex (Mre11-Rad50-Nbs1/Xrs2) resects the $5^{\prime}$ ends to expose $3^{\prime}$ overhangs. Single end invasion (SEI) is mediated by Rad51. Homologous recombination can then proceed through the following pathways: $(A)$ synthesis-dependent strand annealing resulting in noncrossover products or $(B)$ double Holliday junction $(\mathrm{dHJ})$ formation by Mer3 and Msh4-Msh5 resulting in crossover (CO) formation. The DNA helicase ReqQ homologs Sgs1 and RTEL-1 disrupt D-loops to anneal both ends of the DSB. Once double Holliday junctions are formed, they are resolved by the structure-specific endonucleases SLX-1-SLX-4/HIM-18, MUS-81-EME1, and XPF-ERCC1. (C) Asymmetric resolution of the dHJ produces crossovers, and $(D)$ symmetric resolution results in noncrossovers. $(E)$ dHJs can also be processed by the dissolution pathway through the BTR complex (BLM-TOP3-RMI1/2) to make noncrossover products. Paternal DNAs are blue and maternal DNAs are red. Circles indicate the $5^{\prime}$ side of DNA. Orange triangles indicate the direction of catalytic activities of Holliday junction resolvases. Key proteins acting at each step are indicated on the right, and both yeast and worm names are indicated.

one DSB per homologous chromosome pair is sufficient to make a crossover (Rosu et al. 2011). Moreover, chromosomes that fail to undergo crossover formation, as a result of either impaired homologous pairing or, in the case of the extra chromosome present in trisomies, lack of synapsis, are preferentially segregated into the polar bodies during both anaphase I and anaphase II of C. elegans female meiosis (Cortes et al. 2015; Muscat et al. 2015; Vargas et al. 2017). Therefore, crossover formation is not only important for accurate homolog separation at meiosis I but also acts as a driving force during sister chromatid separation at meiosis II.

\section{Crossover Interference}

Crossover interference is a phenomenon in which a crossover at one location reduces the probability of a sec- ond crossover nearby such that when there are two or more crossovers along a bivalent these crossovers are separated away from each other (Fig. 3B). This phenomenon was first described more than 100 years ago (Sturtevant 1915) and is only observed for class I crossover events. A beamfilm model has been proposed for crossover interference that simulates establishment and propagation of a mechanical stress along the chromosome axis depicted by an elastic beam plate (metal) covered with a thin brittle film (ceramic) with crossovers being seen as cracks that release the stress locally and thus abrogate crossovers nearby (Kleckner et al. 2004). Topoisomerase II and the meiosis-specific chromosome axis protein Red1 are suggested to be involved in crossover interference through ubiquitination by the histone deacetylase Sir2 and the SUMOtargeted ubiquitin ligases Slx5-Slx8 (Zhang et al. 2014). Synaptonemal complex-dependent crossover interference 
A

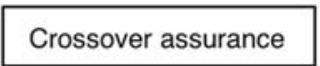

Crossover interference

C

Crossover homeostasis

D

\section{Crossover invariance}

E

Crossover patterning
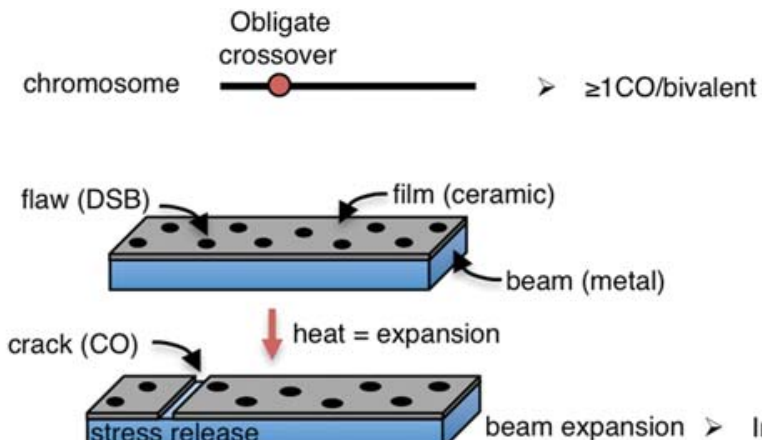

stress release beam expansion > Inhibition of CO nearby

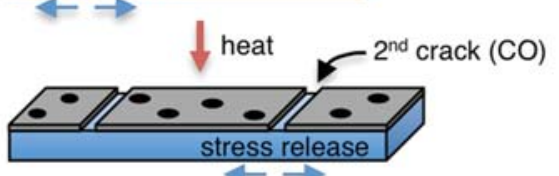

$\rightarrow \rightarrow$

high levels of DSBs low levels of DSBs

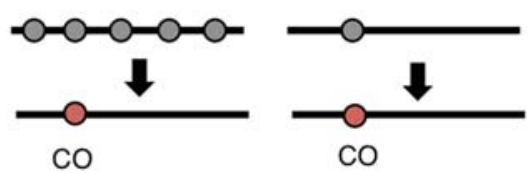

Keep \# of COs constant

$\mathrm{CO}$

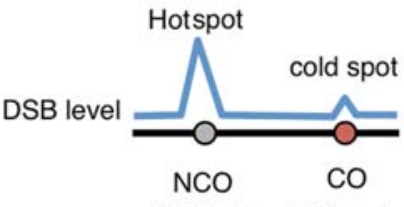

keep the same crossover frequency/ kb (DSB hotspots became CO cold)

(IS bias)

(IH bias)

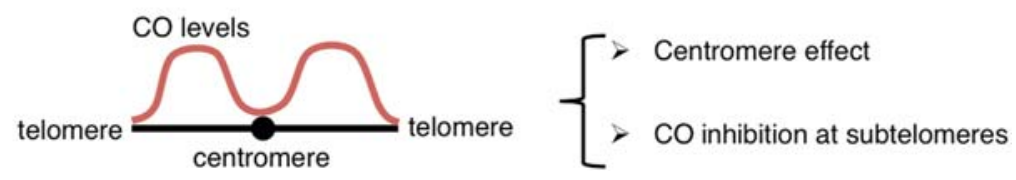

Figure 3. Different types of crossover (CO) control. Five known forms of crossover control are depicted. $(A)$ Crossover assurance. At least one crossover per homologous chromosome pair is essential for chiasma formation and proper chromosome segregation at meiosis I. (B) Crossover interference. The beam-film model (modified from Kleckner et al. 2004) is represented. Chromosome axes and chromatin loops are likened as metallic beams and ceramic films that are tightly bonded to the beam, respectively. Heating the beam results in a flaw (DSB) being converted into a crack (CO formation), and the release of stress then propagates in both directions. Continued heating generates a second crack away from the first crack resembling interference. $(C)$ Crossover homeostasis. Either high or low levels of DSBs per homologous chromosome pair result in the same number of crossovers. Gray circles are DSBs, and orange circles are crossovers. $(D)$ Crossover invariance. DSBs at hotspots tend to undergo intersister bias, resulting in a noncrossover outcome, whereas DSBs at cold spots undergo interhomolog bias leading to crossover products in Schizosaccharomyces pombe. (E) Crossover distribution/centromere effect. Crossovers near centromeres and telomeres are suppressed. Crossovers are also suppressed at the center regions in the holocentric organism Caenorhabditis elegans.

is observed in C. elegans (Libuda et al. 2013) and in at least one yeast strain (Sym and Roeder 1994; Chen et al. 2008). The biological function of crossover interference is largely unknown, but it may confer a selective advantage because of cosegregation of functionally related linked genes (Wang et al. 2015; Sun et al. 2017).

\section{Crossover Homeostasis}

In general, DSBs, which are introduced at the leptotene/ zygotene stage of prophase of meiosis I, occur at levels that are 10-fold higher than the number of crossovers detected. The number of crossovers is maintained constant at the expense of noncrossover events even when DSBs levels are reduced. This phenomenon is termed "crossover homeostasis" and has been observed in yeast (Martini et al. 2006), mice (Cole et al. 2012), worms (Yokoo et al. 2012), and plants (Fig. 3C; Varas et al. 2015).

\section{Crossover Invariance}

The choice of repair template is very important to make an interhomolog crossover. In addition, levels of DSBs vary largely across the genome as evidenced by the presence of both hotspots and cold regions of DSBs along chromosomes. In Schizosaccharomyces pombe, which 
lacks crossover interference, a nearly constant level of crossing-over is maintained per unit physical distance across the genome by control of partner choice for DSB repair, a phenomenon referred to as "crossover invariance" (Fig. 3D; Hyppa and Smith 2010; Fowler et al. 2014). At a DSB hotspot, intersister repair is predominant, whereas at a DSB cold region, interhomolog repair is more prevalent. This phenomenon may serve as an alternative mechanism of crossover homeostasis in other organisms to maintain crossover levels constant.

\section{Crossover Patterning/Centromere Effect}

Crossover formation is inhibited near centromeres and telomeres in many species including humans (Fig. 3E; Ottolini et al. 2015; Ren et al. 2016). Crossovers at centromeres disrupt cohesion in the pericentric region and affect kinetochore orientation. Interestingly, even gene conversion near centromeres is associated with $60 \%$ of segregation errors at meiosis I in Saccharomyces cerevisiae, primarily because of premature sister chromatid separation (Sears et al. 1995). The "centromere effect" was first described in Drosophila as an inhibition of crossovers at centromeres and pericentromeric euchromatic regions (Beadle 1932). A combination of the centromere effect and crossover suppression by the Blm helicase results in the absence of crossovers on chromosome 4 in Drosophila (Hartmann and Sekelsky 2017; Hatkevich and Sekelsky 2017). In plants, cytosines at centromeric regions are highly methylated, thereby suppressing expression of repetitive sequences including transposons. Pollen typing revealed that crossover frequency is increased in centromeric regions in the DNA methyltransferase met 1 mutants, suggesting that DNA methylation at centromeres is important to suppress crossovers at those regions (Yelina et al. 2012). Yeast Slx4, a regulatory subunit of the structure-specific endonuclease Slx 1 , is also required for crossover suppression near centromeres in an Slx1-independent manner (Higashide and Shinohara 2016). A similar suppression mechanism was observed in C. elegans, where normally crossovers are located on the arms but not at the center of the chromosomes (Fig. 4). However, the crossover suppression observed at the center of the chromosomes in this worm is SLX-1-dependent and SLX4/HIM-18-independent (Saito et al. 2009, 2012, 2013). Taken together, these various layers of crossover control underscore the importance of crossover formation during meiosis and the need for tightly regulating this process throughout species.

\section{CROSSOVER PATTERNING IN C. ELEGANS}

Although studies of crossover control started a century ago, the molecular mechanisms underlying these processes are still largely unknown, in part because the various layers of crossover control are not independent phenomena. The regulation of obligatory crossover, interference, and homeostasis are thought to be a cooperative process (Wang et al. 2015). To understand these complex phenomena, it is important to use a simple model.

In C. elegans, crossovers tend to occur in an off-centered position such that crossover frequencies are $1.3 \mathrm{cM} / \mathrm{Mb}$ at the central region of the chromosome, which encompasses

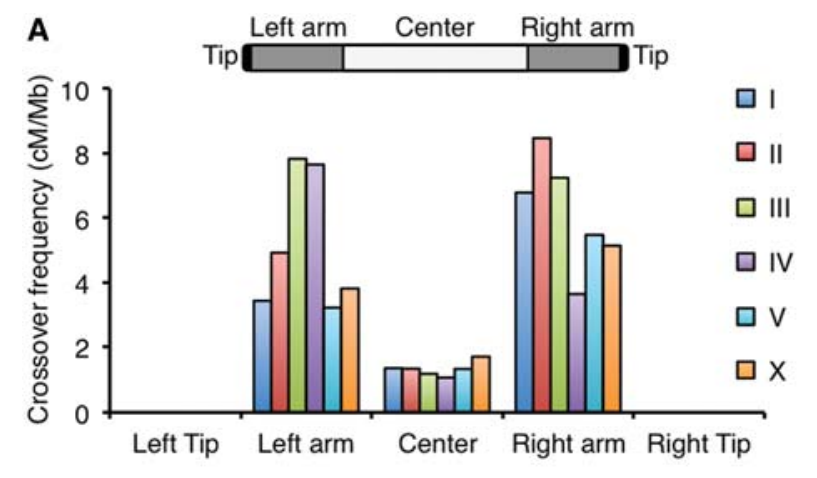

B
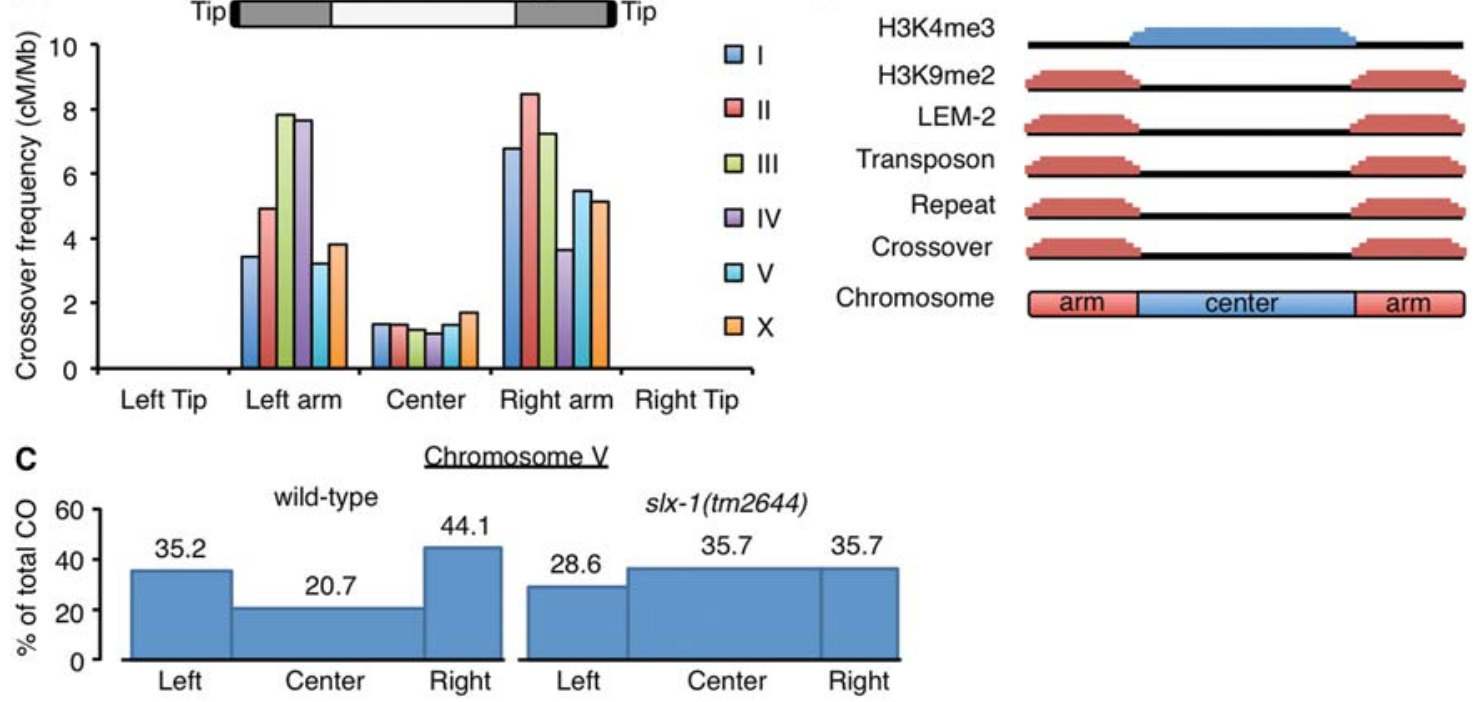

Figure 4. Tight regulation of crossover formation in Caenorhabditis elegans. (A) Crossovers are enriched at arm regions but suppressed at center regions in both autosomes and the $\mathrm{X}$ chromosome in C. elegans. No crossovers are observed at subtelomeric regions (average $<614 \mathrm{~kb}$ from telomeres). Data was adapted from Rockman and Kruglyak (2009). (B) Unique features divide chromosome domains in C. elegans. Although up to approximately 10 DSBs are distributed in a nonbiased manner along chromosomes (Saito et al. 2012), crossovers occur at the arm regions in which the heterochromatin marker histone H3K9me2, the nuclear membrane protein (LEM-2) binding sequences, transposons, and repeat sequences are enriched. Crossover formation is suppressed at the center region in which the euchromatic marker histone $\mathrm{H} 3 \mathrm{~K} 4 \mathrm{me} 3$ is enriched. (C) Crossover suppression at the center region of autosomes is lost in $s / x-1(t m 2644)$ null mutants. Blue boxes indicate crossover frequencies (Saito et al. 2012). Although the overall crossover frequency is not altered, crossover distribution is altered by increasing at the center region and decreasing at the arms in $s l x-1$ mutants compared to wild type. 
$49 \%$ of the total length of the autosomes and $36 \%$ of the X chromosomes, and 4.7 and $6.1 \mathrm{cM} / \mathrm{Mb}$ on the left and right arms, respectively (Fig. 4A; Rockman and Kruglyak 2009). There are some clear differences between the central and arm regions of the chromosomes in this organism. For example, essential genes and the euchromatin marker histone $\mathrm{H} 3 \mathrm{~K} 4 \mathrm{me} 3$ are enriched at the central region. In contrast, the arm regions are gene-poor, they are enriched for the heterochromatin marker $\mathrm{H} 3 \mathrm{~K} 9 \mathrm{me} 2 / 3$, and they are transposon- and repeat-rich and interact with the nuclear membrane via the LEM (LAP2, emerin, MAN1) domaincontaining protein LEM-2 (Fig. 4B; C. elegans Sequencing Consortium 1998; Gerstein et al. 2010; Ikegami et al. 2010; Liu et al. 2011; Ho et al. 2014). Different mutants have been identified that shift crossover distribution to the center region of the chromosomes in C. elegans (Zetka and Rose 1995; Wagner et al. 2010; Meneely et al. 2012; Saito et al. 2012; Chung et al. 2015; Hong et al. 2016; Jagut et al. 2016). However, the molecular mechanism underlying the regulation of crossover position is largely unknown. We found that in mutants for SLX-1, a structure-specific endonuclease that cleaves 5 ' flaps, replication forks, and Holliday junctions, crossovers shifted to the center regions, whereas overall crossover frequency was preserved (Fig. 4C; Saito et al. 2012, 2013). This was not due to alterations in DSB distribution because similar frequencies of markers for DSB repair sites were observed cytologically along the arms and at the center of the chromosomes in slx-1 mutants compared to wild type (Saito et al. 2012). This observation led us to hypothesize that there are mechanisms either inhibiting crossover formation or promoting noncrossover formation after DSB induction at the center region of the chromosomes, such as SDSA, double Holliday junction dissolution, "same sense resolution" of double Holliday junctions, or intersister repair. Based on its Holliday junction resolvase activity, one possibility is that SLX-1 produces noncrossovers at the center region by same sense resolution of double Holliday junctions. An alternative, albeit nonexclusive, possibility is that SLX-1 may act as an epigenetic reader given that it has a PHD/RING-like zinc finger domain implicated in recognizing histone H3K4me3 (Peña et al. 2006; Shi et al. 2006; Matthews et al. 2007; Ramón-Maiques et al. 2007). Given that the euchromatin marker H3K4me3 is enriched at the center region of the autosomes in $C$. elegans, we hypothesize that SLX-1 might be recruited to that region through recognition of H3K4me3 by its PHD/RING finger domain or potentially function as an ubiquitin ligase to activate noncrossover pathways at the center region. It is known that $\mathrm{H} 3 \mathrm{~K} 4 \mathrm{me} 2, \mathrm{H} 3 \mathrm{~K} 4 \mathrm{me} 3$, and H3K9ac are specifically enriched at the DSB hotspots recognized by the PRDM (PRDI-BF1 [positive regulatory domain I-binding factor 1] and RIZ [retinoblastoma-interacting zinc-finger protein] homology domain)-containing histone $\mathrm{H} 3$ methyltransferase Prdm9 in mammals (Hayashi et al. 2005; Buard et al. 2009; Baudat et al. 2010; Myers et al. 2010; Parvanov et al. 2010). After DSBs are introduced, phosphorylation of histone $\mathrm{H} 2 \mathrm{AX}$ and hyperacetylation of histone $\mathrm{H} 4$ are observed near the hotspots (Mahadevaiah et al. 2001; Buard et al. 2009). H3K4me3 is associated with DSB for- mation in yeasts and plants (Choi et al. 2013) and the PHD finger of Spp1, a component of the histone H3K4 methyltransferase Set1 complex (also known as COMPASS), reads $\mathrm{H} 3 \mathrm{~K} 4 \mathrm{me} 3$ to promote DSBs (Acquaviva et al. 2013; Sommermeyer et al. 2013). However, whether $\mathrm{H} 3 \mathrm{~K} 4 \mathrm{me} 3$ is associated with noncrossover pathways and whether crossover hotspots are marked by H3K 9 me $2 / 3$ remain unknown. One possible explanation for why DSBs are introduced at the arm region is that the DSB machinery could access small euchromatic (H3K4me3) sites dispersed throughout the $\mathrm{H} 3 \mathrm{~K} 9 \mathrm{me} 2 / 3$ marked heterochromatin on the arm regions (Ikegami et al. 2010). Combined strategies, described below, allow for direct testing of whether SLX-1 localizes at the center region of chromosomes and the roles of its PHD/RING domain.

\section{INDUCIBLE SINGLE DSB SYSTEM-HOW DOES CROSSOVER POSITION IMPACT CHROMOSOME SEGREGATION IN A METAZOAN?}

Although improper crossover distribution has been detected in aneuploid gametes and embryos in humans, the impact of improper crossover distribution has not been tested directly in metazoans. C. elegans is a suitable model organism to assess how improper crossover distribution can cause errors in meiotic chromosome segregation given how tightly crossovers are regulated in this organism. We previously showed that, starting in late pachytene, chromosomes remodel around the off-centered crossover, resulting in the characteristic cruciform configuration observed for bivalents in late diakinesis consisting of a long and a short arm intersecting at the chiasma (Fig. 5B; Nabeshima et al. 2005). Aurora B kinase, AIR-2, localizes at the short arms by the end of diakinesis in which it phosphorylates the meiosis-specific cohesin REC-8. This triggers degradation of sister chromatid cohesion along the short arm. LAB-1, a functional shugoshin analog, antagonizes AIR-2 along the long arms, thereby protecting these chromosome subdomains from premature loss of sister chromatid cohesion. Therefore, we hypothesized that achieving proper chromosome remodeling must be critical for an accurate reductional division at meiosis I. To test this directly, we used a single DSB-inducible system in which the Mos 1 transposon from Drosophila was introduced into $C$. elegans along with a transposase under a heat shock promoter and a spo-11 mutation that eliminates endogenous meiotic DSBs (Bessereau et al. 2001). We obtained lines in which a single DSB was produced at either the physical center or at the subtelomeric region in an autosome and compared that to lines in which a single DSB was produced at an off-centered position (Fig. 5A,B). We observed impaired chromosome remodeling based on mislocalization of AIR-2 and LAB-1 as well as subsequent increased nondisjunction when the single DSB was converted into a crossover at the center position. We did not observe a single crossover event at the subtelomeric position although a DSB at such a position did get designated into a crossover, suggesting 
A

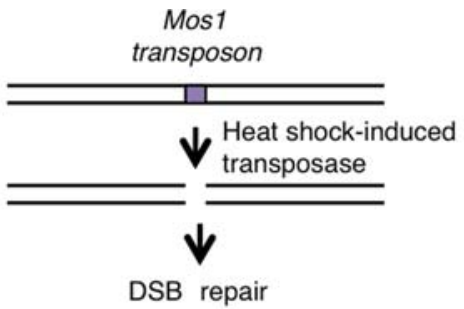

B

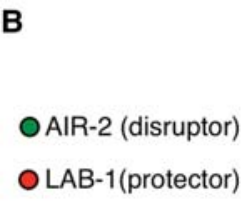

$\mathrm{CO}$ at off-centered position
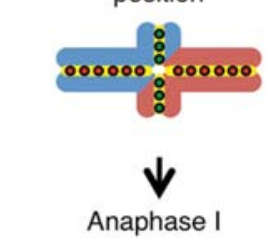

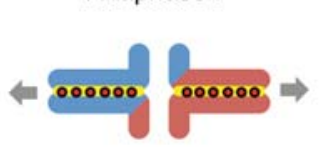

Figure 5. Site-specific analysis of meiotic recombination in Caenorhabditis elegans. (A) Mos1-based single inducible double-strand break (DSB) system. Mos 1 transposons and transposases are integrated into chromosomes in a spo-11 mutant background. The Mos 1 integrated strain library is available at NemaGENETAG (http://elegans.imbb.forth.gr/nemagenetag/). Heat shock induces expression of the transposase, which excises the Mos 1 transposon resulting in a single DSB at a specific genomic position. $(B)$ The single DSB system can be used to investigate the positional effect of crossovers on meiotic chromosome segregation. Blue and pink lines are paternal and maternal chromatids, respectively. The system allows us to analyze the outcome of a single crossover (CO) forming at specific chromosomal sites. A crossover at the very center region disrupts the asymmetric configuration of the bivalent, resulting in premature sister chromatid separation or homolog nondisjunction. Crossovers at subtelomeres result either in potentially fragile connections that are not stably retained at the ends of very short arms or events that fail to mature into crossovers at those positions.

these either cannot be stably maintained or are not effectively processed into a mature crossover at that position (E Altendorfer and MP Colaiácovo, unpubl.). This is supported by the lack of crossovers previously reported at subtelomeric regions by Rockman and Kruglyak (2009). In contrast, a DSB at an off-centered position proceeded as wild type. We are currently assessing whether bivalent formation is increased if a single DSB is induced at the center region of the chromosomes in slx-1 mutants to confirm our genetic observation that SLX-1 suppresses crossovers at that region. This is being combined with ChIP-seq for epigenetic markers with the goal of unveiling how crossover position impacts chromosome remodeling and proper chromosome segregation and how structurespecific endonucleases are involved in this regulation.

\section{IS THE CLASS II CROSSOVER PATHWAY CONSERVED IN C. ELEGANS?}

It has been proposed that only class I crossovers exist in C. elegans, because almost no crossovers are detected in worm mutants of the $z m m$ counterparts (syp-1, syp-2, syp3, syp-4, zhp-3, him-14/msh4, msh-5) (Zalevsky et al. 1999; Kelly et al. 2000; MacQueen et al. 2002; Colaiácovo et al. 2003; Jantsch et al. 2004; Smolikov et al. 2007, 2009) and crossover interference is extremely robust given that only a single crossover occurs per bivalent in normal conditions (Brenner 1974; Barnes et al. 1995). Whereas in budding yeast, the structure-specific endonucleases Slx1-
Slx4 and Mus81-Mms4 are required for class II crossovers (Zakharyevich et al. 2012), in C. elegans SLX-1HIM-18/SLX4 and MUS-81 appear to be involved in $20 \%-60 \%$ of total crossovers together with XPF- 1 , another structure-specific endonuclease (Saito et al. 2009, 2012, 2013; Agostinho et al. 2013; O’Neil et al. 2013).

Interestingly, class II crossovers are observed in C. elegans under conditions in which there is either an excess of DSBs or a mutation in the antirecombinase RTEL-1 helicase (Youds et al. 2010). DSBs induced artificially by $\gamma$-irradiation (IR) cause a dose-dependent (10 and $75 \mathrm{~Gy}$ ) increase in the number of crossovers, whereas the number of crossover-designated sites marked by ZHP3 is kept at normal levels after IR treatment (Youds et al. 2010). Moreover, these excess crossovers are suppressed back to wild-type levels in mus-81 mutants (Youds et al. 2010). Therefore, there must be designation-independent and MUS81-dependent class II crossovers in C. elegans as well. Furthermore, crossovers in rtel-1 mutants are randomly distributed, suggesting that RTEL-1-inhibited crossovers show no interference (Youds et al. 2010). Helicase-dependent inhibition of class II crossovers is conserved in budding yeast where it is exerted by Sgs1 (De Muyt et al. 2012; Zakharyevich et al. 2012), in fission yeast by Fml1 (Lorenz et al. 2012), and in plants by FANCM (Crismani et al. 2012; Knoll et al. 2012). These data and the wide conservation of class II crossovers in yeast (de los Santos et al. 2003), plants (Berchowitz et al. 2007; Higgins et al. 2008), and mammals (Holloway et al. 
2008; Svetlanov et al. 2008) suggest that a "dormant" class II crossover pathway exists in C. elegans.

Mapping of crossover frequency and distribution in either IR-treated combinatorial mutants of structure-specific endonucleases or in mutants for the structure-specific endonucleases combined with an rtel-1 mutation will reveal whether interference-independent class II crossovers are structure-specific endonuclease-dependent as well in C. elegans.

\section{SEXUAL DIMORPHISM IN CROSSOVER REGULATION DURING C. ELEGANS MEIOSIS}

The biggest difference regarding crossover control between sexes is observed in Drosophila, in which crossovers occur in females but not in males. However, differences are observed in other organisms as well. For example, although the number of crossovers seems similar between female and male meiosis in mice, based on the number of MLH1 foci (females $=23.0$ and males $=22.7$ ) (Holloway et al. 2008), high-resolution sex-specific linkage maps revealed that crossover frequency on autosomes in females $(\sim 71 \mathrm{cM} /$ autosome $)$ is higher than in males ( $\sim 66 \mathrm{cM} /$ autosome) (Liu et al. 2014). Interestingly, the genomic distribution of crossovers between female and male meiosis is also significantly different; crossover distributions are uniform in females, but subtelomerically enhanced and pericentromerically suppressed in males (Liu et al. 2014). In contrast, opposite results regarding crossover frequency were observed in plants, in which male crossover frequency (2.23 crossovers/bivalent) is 1.7 -fold higher than in females (1.33 crossovers/bivalent) (Giraut et al. 2011), even though the distribution of crossovers follows a similar pattern to that observed in mice. Specifically, crossover frequencies at subtelomeric regions are very high in males and very low in females (Giraut et al. 2011). In human females, the number of MLH1 foci is greater than in males, whereas the average crossover frequency is similar between the sexes (Wang et al. 2015). This suggests that crossover maturation after designation may be less efficient in females than in males. In C. elegans, crossover frequency is higher in male spermatogenesis than in oogenesis. The ratio of double crossovers is $\sim 4 \%$ at chromosomes IV and $\mathrm{V}$ in males, whereas almost no double crossovers are detected in oogenesis (Henzel et al. 2011; Gabdank and Fire 2014). Furthermore, him- 8 and meDf2 mutants, which lack crossovers on the X chromosome because of impaired X chromosome synapsis, show a higher crossover frequency (including double crossovers) on the autosomes compared with wild type (Carlton et al. 2006). These observations suggest that a crossover homeostasis-like regulation that maintains the crossover number per "nucleus" (referred to as an interchromosomal effect [Sturtevant 1919; Lucchesi and Suzuki 1968]) exists in animals that have an XO sex type such as C. elegans males as well. How the "additional" crossovers are formed/allowed is an interesting question. The following aspects should be considered: (1) Crossover interference is stronger in hermaphrodite oogenesis than in male spermatogenesis in C. elegans (Gab- dank and Fire 2014); and (2) surveillance mechanisms to check the number and distribution of crossovers (crossover checkpoint) may be weak in XO animals. In fact, although either accumulated DNA damage or aberrant synapsis induces apoptosis resulting in the removal of the affected cell in hermaphrodite gonads, there is no germline apoptosis in C. elegans males because of lack of CED-3 activation in the male germline even though a recombination checkpoint is activated in male gonads (JaramilloLambert et al. 2010). Whether there are also differences in crossover control between spermatogenesis and oogenesis in hermaphrodites remains to be investigated.

\section{CROSSOVER REGULATION IN AUTOSOMES AND SEX CHROMOSOMES}

Crossover regulation is different between autosomes and sex chromosomes. $\mathrm{X}$ and $\mathrm{Y}$ chromosomes in mammals, including humans, pair via short homologous sequences referred to as the pseudoautosomal region (PAR) located at their subtelomeres. C. elegans provides a useful experimental system to understand the evolution of heteromorphic sex chromosomes (Henzel et al. 2011). The karyotype of the C. elegans hermaphrodite consists of five autosomes (5A) and XX, and the male of $5 \mathrm{~A}$ and XO. An end-to-end fusion of chromosomes $\mathrm{X}$ and IV generates a new chromosome named $m n T 12$, and males with $m n T 12$ form a neo-sex body (IV $=$ neo- $Y$ and IV-X fusion $=$ neo$\mathrm{X}$ ), in which a portion of chromosome IV mimics the mammalian PAR during meiotic prophase (Sigurdson et al. 1986). Meiotic sex chromosome inactivation (MSCI) occurs during meiotic prophase, and several factors have been identified as required for mammalian MSCI such as $\gamma \mathrm{H} 2 \mathrm{AX}$, ATR kinase, ubiquitin, SUMO, and the BRCA1-A complex (Turner 2007; Lu and $\mathrm{Yu}$ 2015). However, the links between MSCI and crossover control remain to be determined. Whether MCSI is conserved in the C. elegans neo-sex body also requires further investigation. The unsynapsed $\mathrm{X}$ chromosome region of the neo-sex body in C. elegans males harbors high levels of the heterochromatic marker $\mathrm{H} 3 \mathrm{~K} 9 \mathrm{me} 2$, similar to the $\mathrm{X}-\mathrm{Y}$ bodies in mammals (Henzel et al. 2011). The PAR must receive DSBs to make an obligate crossover like other autosomes. In mice, chromatin axis length at the PAR is long relative to DNA length and in contrast to autosome axes in which DNA content correlates well with axis length (Kauppi et al. 2011). This was proposed to result in shorter chromatin loops on the PAR and contribute to higher DSB levels at this region compared to autosomes. Longer axes are also observed along the paired chromosome IV and mnT12 in C. elegans (Henzel et al. 2011). Because Spo11 $\alpha$-dependent induction of DSBs is observed at the PAR in mice (Kauppi et al. 2011), it would be interesting to investigate whether there are differences between the paired chromosome IV and $m n T 12$ and other normal chromosome pairs regarding the machinery being engaged for DSB formation.

DSBs are also induced at the nonhomologous regions of sex bodies in mammals and on the $\mathrm{X}$ chromosome in males in C. elegans that lack a homologous partner. The 
biological function for DSB repair in these cases is unknown, but interestingly, DSBs on the male X chromosome may be partially inhibited by the structure-specific endonuclease XPF-1 in C. elegans (Checchi et al. 2014). Another possible explanation is that XPF-1 functions for repair via single-strand annealing at repeat sequences when RAD-51-dependent homologous recombination is not available on male $\mathrm{X}$ chromosomes (Checchi et al. 2014). Further studies are required to understand how DSBs are induced and repaired in XO animals.

In the $C$. elegans hermaphrodite, $0.2 \%$ of its progeny are males resulting from $\mathrm{X}$ chromosome nondisjunctions during meiosis. Based on the observations that most autosomal aneuploidies are lethal and that nearly $100 \%$ of the embryos hatch during hermaphrodite reproduction, $\mathrm{X}$ chromosomes in C. elegans are more vulnerable to disjoining during meiosis compared with autosomes. We reported that combinatorial mutants of different structurespecific endonucleases caused higher reductions in crossover frequencies on $\mathrm{X}$ chromosomes than on autosomes (Saito et al. 2009, 2012, 2013). Understanding the fragility of the X chromosome in the context of crossover control is important. Several known differences were reported between the $\mathrm{X}$ and the autosomes. First, the right third of the $\mathrm{X}$ chromosome has fewer heterochromatin marks such as $\mathrm{H} 3 \mathrm{~K} 9 \mathrm{me} 2 / 3$ and nuclear membrane binding regions compared to the autosomes (Ikegami et al. 2010; Liu et al. 2011). Second, the $X$ chromosome undergoes fewer DSBs than the autosomes (Gao et al. 2015). Further, both the timing of replication and the onset and completion of synapsis are delayed for the $\mathrm{X}$ chromosome in C. elegans (Jaramillo-Lambert et al. 2007; Mlynarczyk-Evans and Villeneuve 2017). In yeasts and plants, the regulation of DNA replication and induction of meiotic DSBs are well connected (Murakami and Nurse 2001; Higgins et al. 2012; Murakami and Keeney 2014). Whether the delay in replication is related to DSB induction on the X chromosomes in $C$. elegans remains to be analyzed. Now we can trace the fate of specific numbers and locations of DSBs by using an inducible single DSB system in C. elegans (Fig. 5A,B). Use of this system, in combination with high-resolution microscopy, single-nucleotide polymorphism (SNP) mapping, and sequence-based genomics approaches, will allow us to address the remaining questions described above.

\section{CONCLUSION}

Although crossover control has been a topic of extensive studies, the molecular mechanisms underlying its regulation are largely unknown. Technological advancements now allow for the introduction and analysis of a single (or more) crossover at specific genomic positions in metazoans. In addition to Mos 1 excision, a CRISPR (clustered regularly interspaced short palindromic repeat)-based DSB induction system will be established shortly. These approaches will unveil the positional effects of crossovers, allowing us to understand the origin of aneuploidies. This in turn may have clinical repercussions for treatments of infertility and in finding targets for cancer therapy in humans.

\section{ACKNOWLEDGMENTS}

We thank Marina Martinez Garcia for critical reading of this manuscript. This work was supported by the National Institutes of Health grant R01GM105853 to M.P.C. We apologize to authors whose work was not cited because of space constraints.

\section{REFERENCES}

Acquaviva L, Székvölgyi L, Dichtl B, Dichtl BS, de La Roche Saint André C, Nicolas A, Géli V. 2013. The COMPASS subunit Spp1 links histone methylation to initiation of meiotic recombination. Science 339: 215-218.

Agostinho A, Meier B, Sonneville R, Jagut M, Woglar A, Blow J, Jantsch V, Gartner A. 2013. Combinatorial regulation of meiotic Holliday junction resolution in $C$. elegans by HIM-6 (BLM) helicase, SLX-4, and the SLX-1, MUS-81 and XPF1 nucleases. PLoS Genet 9: e1003591.

Barnes TM, Kohara Y, Coulson A, Hekimi S. 1995. Meiotic recombination, noncoding DNA and genomic organization in Caenorhabditis elegans. Genetics 141: 159-179.

Baudat F, Buard J, Grey C, Fledel-Alon A, Ober C, Przeworski M, Coop G, de Massy B. 2010. PRDM9 is a major determinant of meiotic recombination hotspots in humans and mice. Science 327: 836-840.

Beadle GW. 1932. A possible influence of the spindle fibre on crossing-over in Drosophila. Proc Natl Acad Sci 18: 160-165.

Berchowitz LE, Francis KE, Bey AL, Copenhaver GP. 2007. The role of AtMUS81 in interference-insensitive crossovers in A. thaliana. PLoS Genet 3: e132.

Bessereau JL, Wright A, Williams DC, Schuske K, Davis MW, Jorgensen EM. 2001. Mobilization of a Drosophila transposon in the Caenorhabditis elegans germ line. Nature 413: 70-74.

Brenner S. 1974. The genetics of Caenorhabditis elegans. Genetics 77: 71-94.

Buard J, Barthes P, Grey C, de Massy B. 2009. Distinct histone modifications define initiation and repair of meiotic recombination in the mouse. EMBO J 28: 2616-2624.

Carlton PM, Farruggio AP, Dernburg AF. 2006. A link between meiotic prophase progression and crossover control. PLoS Genet 2: e12.

C. elegans Sequencing Consortium. 1998. Genome sequence of the nematode C. elegans: A platform for investigating biology. Science 282: 2012-2018.

Checchi PM, Lawrence KS, Van MV, Larson BJ, Engebrecht J. 2014. Pseudosynapsis and decreased stringency of meiotic repair pathway choice on the hemizygous sex chromosome of Caenorhabditis elegans males. Genetics 197: 543-560.

Chen SY, Tsubouchi T, Rockmill B, Sandler JS, Richards DR, Vader G, Hochwagen A, Roeder GS, Fung JC. 2008. Global analysis of the meiotic crossover landscape. Dev Cell 15: 401415.

Choi K, Zhao X, Kelly KA, Venn O, Higgins JD, Yelina NE, Hardcastle TJ, Ziolkowski PA, Copenhaver GP, Franklin FC, et al. 2013. Arabidopsis meiotic crossover hot spots overlap with H2A.Z nucleosomes at gene promoters. Nat Genet 45: 1327-1336.

Chua PR, Roeder GS. 1998. Zip2, a meiosis-specific protein required for the initiation of chromosome synapsis. Cell 93: 349-359.

Chung G, Rose AM, Petalcorin MI, Martin JS, Kessler Z, Sanchez-Pulido L, Ponting CP, Yanowitz JL, Boulton SJ. 2015. REC-1 and HIM-5 distribute meiotic crossovers and function redundantly in meiotic double-strand break formation in $\mathrm{Cae}$ norhabditis elegans. Genes Dev 29: 1969-1979. 
Colaiácovo MP, MacQueen AJ, Martinez-Perez E, McDonald K, Adamo A, La Volpe A, Villeneuve AM. 2003. Synaptonemal complex assembly in C. elegans is dispensable for loading strand-exchange proteins but critical for proper completion of recombination. Dev Cell 5: 463-474.

Cole F, Kauppi L, Lange J, Roig I, Wang R, Keeney S, Jasin M. 2012. Homeostatic control of recombination is implemented progressively in mouse meiosis. Nat Cell Biol 14: 424-430.

Cooper KW. 1949. The cytogenetics of meiosis in Drosophila; mitotic and meiotic autosomal chiasmata without crossing over in the male. J Morphol 84: 81-121.

Cortes DB, McNally KL, Mains PE, McNally FJ. 2015. The asymmetry of female meiosis reduces the frequency of inheritance of unpaired chromosomes. Elife 4: e06056.

Crismani W, Girard C, Froger N, Pradillo M, Santos JL, Chelysheva L, Copenhaver GP, Horlow C, Mercier R. 2012. FANCM limits meiotic crossovers. Science 336: 1588-1590.

de los Santos T, Hunter N, Lee C, Larkin B, Loidl J, Hollingsworth NM. 2003. The Mus81/Mms4 endonuclease acts independently of double-Holliday junction resolution to promote a distinct subset of crossovers during meiosis in budding yeast. Genetics 164: 81-94.

De Muyt A, Jessop L, Kolar E, Sourirajan A, Chen J, Dayani Y, Lichten M. 2012. BLM helicase ortholog Sgs1 is a central regulator of meiotic recombination intermediate metabolism. Mol Cell 46: 43-53.

Fowler KR, Sasaki M, Milman N, Keeney S, Smith GR. 2014. Evolutionarily diverse determinants of meiotic DNA break and recombination landscapes across the genome. Genome Res 24: 1650-1664.

Gabdank I, Fire AZ. 2014. Gamete-type dependent crossover interference levels in a defined region of Caenorhabditis elegans chromosome V. G3 (Bethesda) 4: 117-120.

Gao J, Kim HM, Elia AE, Elledge SJ, Colaiácovo MP. 2015. NatB domain-containing CRA-1 antagonizes hydrolase ACER-1 linking acetyl-CoA metabolism to the initiation of recombination during C. elegans meiosis. PLoS Genet 11: e1005029.

Gerstein MB, Lu ZJ, Van Nostrand EL, Cheng C, Arshinoff BI, Liu T, Yip KY, Robilotto R, Rechtsteiner A, Ikegami K, et al. 2010. Integrative analysis of the Caenorhabditis elegans genome by the modENCODE project. Science 330: 1775-1787.

Giraut L, Falque M, Drouaud J, Pereira L, Martin OC, Mézard C. 2011. Genome-wide crossover distribution in Arabidopsis thaliana meiosis reveals sex-specific patterns along chromosomes. PLoS Genet 7: e1002354.

Hartmann MA, Sekelsky J. 2017. The absence of crossovers on chromosome 4 in Drosophila melanogaster: Imperfection or interesting exception? Fly (Austin) 20: 1-7.

Hatkevich T, Sekelsky J. 2017. Bloom syndrome helicase in meiosis: Pro-crossover functions of an anti- crossover protein. Bioessays 39. doi: 10.1002/bies.201700073.

Hayashi K, Yoshida K, Matsui Y. 2005. A histone H3 methyltransferase controls epigenetic events required for meiotic prophase. Nature 438: 374-378.

Henzel JV, Nabeshima K, Schvarzstein M, Turner BE, Villeneuve AM, Hillers KJ. 2011. An asymmetric chromosome pair undergoes synaptic adjustment and crossover redistribution during Caenorhabditis elegans meiosis: Implications for sex chromosome evolution. Genetics 187: 685-699.

Higashide M, Shinohara M. 2016. Budding yeast SLX4 contributes to the appropriate distribution of crossovers and meiotic double-strand break formation on bivalents during meiosis. $G 3$ (Bethesda) 6: 2033-2042.

Higgins JD, Buckling EF, Franklin FC, Jones GH. 2008. Expression and functional analysis of AtMUS81 in Arabidopsis meiosis reveals a role in the second pathway of crossing-over. Plant J 54: 152-162.

Higgins JD, Perry RM, Barakate A, Ramsay L, Waugh R, Halpin C, Armstrong SJ, Franklin FC. 2012. Spatiotemporal asymmetry of the meiotic program underlies the predominantly distal distribution of meiotic crossovers in barley. Plant Cell 24: 4096-4109.
Ho JW, Jung YL, Liu T, Alver BH, Lee S, Ikegami K, Sohn KA, Minoda A, Tolstorukov MY, Appert A, et al. 2014. Comparative analysis of metazoan chromatin organization. Nature 512: 449-452.

Holloway JK, Booth J, Edelmann W, McGowan CH, Cohen PE. 2008. MUS81 generates a subset of MLH1-MLH3-independent crossovers in mammalian meiosis. PLoS Genet 4: e1000186.

Hong Y, Sonneville R, Agostinho A, Meier B, Wang B, Blow JJ, Gartner A. 2016. The SMC-5/6 complex and the HIM-6 (BLM) helicase synergistically promote meiotic recombination intermediate processing and chromosome maturation during Caenorhabditis elegans meiosis. PLoS Genet 12: e1005872.

Hyppa RW, Smith GR. 2010. Crossover invariance determined by partner choice for meiotic DNA break repair. Cell 142: 243-255.

Ikegami K, Egelhofer TA, Strome S, Lieb JD. 2010. Caenorhabditis elegans chromosome arms are anchored to the nuclear membrane via discontinuous association with LEM-2. Genome Biol 11: R120.

Jagut M, Hamminger P, Woglar A, Millonigg S, Paulin L, Mikl M, Dello Stritto MR, Tang L, Habacher C, Tam A, et al. 2016. Separable roles for a Caenorhabditis elegans RMI1 homolog in promoting and antagonizing meiotic crossovers ensure faithful chromosome inheritance. PLoS Biol 14: e1002412.

Jantsch V, Pasierbek P, Mueller MM, Schweizer D, Jantsch M, Loidl J. 2004. Targeted gene knockout reveals a role in meiotic recombination for ZHP-3, a Zip3-related protein in Caenorhabditis elegans. Mol Cell Biol 24: 7998-8006.

Jaramillo-Lambert A, Ellefson M, Villeneuve AM, Engebrecht J. 2007. Differential timing of S phases, X chromosome replication, and meiotic prophase in the C. elegans germ line. Dev Biol 308: 206-221.

Jaramillo-Lambert A, Harigaya Y, Vitt J, Villeneuve A, Engebrecht J. 2010. Meiotic errors activate checkpoints that improve gamete quality without triggering apoptosis in male germ cells. Curr Biol 20: 2078-2089.

Kauppi L, Barchi M, Baudat F, Romanienko PJ, Keeney S, Jasin M. 2011. Distinct properties of the XY pseudoautosomal region crucial for male meiosis. Science 331: 916-920.

Keeney S, Giroux CN, Kleckner N. 1997. Meiosis-specific DNA double-strand breaks are catalyzed by Spo11, a member of a widely conserved protein family. Cell 88: $375-384$.

Kelly KO, Dernburg AF, Stanfield GM, Villeneuve AM. 2000. Caenorhabditis elegans msh-5 is required for both normal and radiation-induced meiotic crossing over but not for completion of meiosis. Genetics 156: 617-630.

Kleckner N, Zickler D, Jones GH, Dekker J, Padmore R, Henle J, Hutchinson J. 2004. A mechanical basis for chromosome function. Proc Natl Acad Sci 101: 12592-12597.

Knoll A, Higgins JD, Seeliger K, Reha SJ, Dangel NJ, Bauknecht M, Schropfer S, Franklin FC, Puchta H. 2012. The Fanconi anemia ortholog FANCM ensures ordered homologous recombination in both somatic and meiotic cells in Arabidopsis. Plant Cell 24: 1448-1464.

Libuda DE, Uzawa S, Meyer BJ, Villeneuve AM. 2013. Meiotic chromosome structures constrain and respond to designation of crossover sites. Nature 502: 703-706.

Liu T, Rechtsteiner A, Egelhofer TA, Vielle A, Latorre I, Cheung MS, Ercan S, Ikegami K, Jensen M, Kolasinska-Zwierz P, et al. 2011. Broad chromosomal domains of histone modification patterns in C. elegans. Genome Res 21: 227-236.

Liu EY, Morgan AP, Chesler EJ, Wang W, Churchill GA, PardoManuel de Villena F. 2014. High-resolution sex-specific linkage maps of the mouse reveal polarized distribution of crossovers in male germline. Genetics 197: 91-106.

Lorenz A, Osman F, Sun W, Nandi S, Steinacher R, Whitby MC. 2012. The fission yeast FANCM ortholog directs non-crossover recombination during meiosis. Science 336: 1585-1588.

Lu LY, Yu X. 2015. Double-strand break repair on sex chromosomes: Challenges during male meiotic prophase. Cell Cycle 14: $516-525$. 
Lucchesi J, Suzuki DT. 1968. The interchromosomal control of recombination. Annu Rev Genet 2: 53-86.

Macaisne N, Novatchkova M, Peirera L, Vezon D, Jolivet S, Froger N, Chelysheva L, Grelon M, Mercier R. 2008. SHOC1, an XPF endonuclease-related protein, is essential for the formation of class I meiotic crossovers. Curr Biol 18: 14321437.

MacQueen AJ, Colaiácovo MP, McDonald K, Villeneuve AM. 2002. Synapsis-dependent and -independent mechanisms stabilize homolog pairing during meiotic prophase in C. elegans. Genes Dev 16: 2428-2442.

Mahadevaiah SK, Turner JM, Baudat F, Rogakou EP, de Boer P, Blanco-Rodríguez J, Jasin M, Keeney S, Bonner WM, Burgoyne PS. 2001. Recombinational DNA double-strand breaks in mice precede synapsis. Nat Genet 27: 271-276.

Martinez-Perez E, Colaiácovo MP. 2009. Distribution of meiotic recombination events: Talking to your neighbors. Curr Opin Genet Dev 19: 105-112.

Martini E, Diaz RL, Hunter N, Keeney S. 2006. Crossover homeostasis in yeast meiosis. Cell 126: 285-295.

Matthews AG, Kuo AJ, Ramón-Maiques S, Han S, Champagne KS, Ivanov D, Gallardo M, Carney D, Cheung P, Ciccone DN, et al. 2007. RAG2 PHD finger couples histone H3 lysine 4 trimethylation with V(D)J recombination. Nature 450: 1106 1110 .

Mazina OM, Mazin AV, Nakagawa T, Kolodner RD, Kowalczykowski SC. 2004. Saccharomyces cerevisiae Mer3 helicase stimulates 3'-5' heteroduplex extension by Rad51; implications for crossover control in meiotic recombination. Cell 117: 47-56.

Meneely PM, McGovern OL, Heinis FI, Yanowitz JL. 2012. Crossover distribution and frequency are regulated by him-5 in Caenorhabditis elegans. Genetics 190: 1251-1266.

Mlynarczyk-Evans S, Villeneuve AM. 2017. Time-course analysis of early meiotic prophase events informs mechanisms of homolog pairing and synapsis in Caenorhabditis elegans. Genetics 207: 103-114.

Murakami H, Keeney S. 2014. DDK links replication and recombination in meiosis. Cell Cycle 13: 3621-3622.

Murakami H, Nurse P. 2001. Regulation of premeiotic S phase and recombination-related double-strand DNA breaks during meiosis in fission yeast. Nat Genet 28: 290-293.

Muscat CC, Torre-Santiago KM, Tran MV, Powers JA, Wignall SM. 2015. Kinetochore-independent chromosome segregation driven by lateral microtubule bundles. Elife 4: e06462.

Myers S, Bowden R, Tumian A, Bontrop RE, Freeman C, MacFie TS, McVean G, Donnelly P. 2010. Drive against hotspot motifs in primates implicates the PRDM9 gene in meiotic recombination. Science 327: 876-879.

Nabeshima K, Villeneuve AM, Colaiácovo MP. 2005. Crossing over is coupled to late meiotic prophase bivalent differentiation through asymmetric disassembly of the SC. J Cell Biol 168: $683-689$.

Nakagawa T, Ogawa H. 1999. The Saccharomyces cerevisiae $M E R 3$ gene, encoding a novel helicase-like protein, is required for crossover control in meiosis. EMBO J 18: 5714-5723.

O’Neil NJ, Martin JS, Youds JL, Ward JD, Petalcorin MI, Rose AM, Boulton SJ. 2013. Joint molecule resolution requires the redundant activities of MUS-81 and XPF-1 during Caenorhabditis elegans meiosis. PLoS Genet 9: e1003582.

Ottolini CS, Newnham L, Capalbo A, Natesan SA, Joshi HA, Cimadomo D, Griffin DK, Sage K, Summers MC, Thornhill $\mathrm{AR}$, et al. 2015. Genome-wide maps of recombination and chromosome segregation in human oocytes and embryos show selection for maternal recombination rates. Nat Genet 47: 727-735.

Parvanov ED, Petkov PM, Paigen K. 2010. Prdm9 controls activation of mammalian recombination hotspots. Science 327: 835.

Peña PV, Davrazou F, Shi X, Walter KL, Verkhusha VV, Gozani O, Zhao R, Kutateladze TG. 2006. Molecular mechanism of histone $\mathrm{H} 3 \mathrm{~K} 4 \mathrm{me} 3$ recognition by plant homeodomain of ING2. Nature 442: 100-103.
Ramón-Maiques S, Kuo AJ, Carney D, Matthews AG, Oettinger MA, Gozani O, Yang W. 2007. The plant homeodomain finger of RAG2 recognizes histone $\mathrm{H} 3$ methylated at both lysine-4 and arginine-2. Proc Natl Acad Sci 104: 18993-18998.

Ren H, Ferguson K, Kirkpatrick G, Vinning T, Chow V, Ma S. 2016. Altered crossover distribution and frequency in spermatocytes of infertile men with azoospermia. PLoS One 11: $\mathrm{e} 0156817$.

Rockman MV, Kruglyak L. 2009. Recombinational landscape and population genomics of Caenorhabditis elegans. PLoS Genet 5: e1000419.

Rosu S, Libuda DE, Villeneuve AM. 2011. Robust crossover assurance and regulated interhomolog access maintain meiotic crossover number. Science 334: 1286-1289.

Saito TT, Youds JL, Boulton SJ, Colaiácovo MP. 2009. Caenorhabditis elegans HIM-18/SLX-4 interacts with SLX-1 and XPF-1 and maintains genomic integrity in the germline by processing recombination intermediates. PLoS Genet 5: e1000735.

Saito TT, Mohideen F, Meyer K, Harper JW, Colaiácovo MP. 2012. SLX-1 is required for maintaining genomic integrity and promoting meiotic noncrossovers in the Caenorhabditis elegans germline. PLoS Genet 8: e1002888.

Saito TT, Lui DY, Kim HM, Meyer K, Colaiácovo MP. 2013. Interplay between structure-specific endonucleases for crossover control during Caenorhabditis elegans meiosis. PLoS Genet 9: e1003586.

Sears DD, Hegemann JH, Shero JH, Hieter P. 1995. Cis-acting determinants affecting centromere function, sister-chromatid cohesion and reciprocal recombination during meiosis in $\mathrm{Sac}$ charomyces cerevisiae. Genetics 139: 1159-1173.

Shi X, Hong T, Walter KL, Ewalt M, Michishita E, Hung T, Carney D, Peña P, Lan F, Kaadige MR, et al. 2006. ING2 PHD domain links histone H3 lysine 4 methylation to active gene repression. Nature 442: 96-99.

Shinohara M, Oh SD, Hunter N, Shinohara A. 2008. Crossover assurance and crossover interference are distinctly regulated by the ZMM proteins during yeast meiosis. Nat Genet 40: 299309.

Sigurdson DC, Herman RK, Horton CA, Kari CK, Pratt SE. 1986. An X-autosome fusion chromosome of Caenorhabditis elegans. Mol Gen Genet 202: 212-218.

Smolikov S, Eizinger A, Hurlburt A, Rogers E, Villeneuve AM, Colaiácovo MP. 2007. Synapsis-defective mutants reveal a correlation between chromosome conformation and the mode of double-strand break repair during Caenorhabditis elegans meiosis. Genetics 176: 2027-2033.

Smolikov S, Schild-Prüfert K, Colaiácovo MP. 2009. A yeast two-hybrid screen for SYP-3 interactors identifies SYP-4, a component required for synaptonemal complex assembly and chiasma formation in Caenorhabditis elegans meiosis. PLoS Genet 5: e1000669.

Snowden T, Acharya S, Butz C, Berardini M, Fishel R. 2004. hMSH4-hMSH5 recognizes Holliday junctions and forms a meiosis-specific sliding clamp that embraces homologous chromosomes. Mol Cell 15: 437-451.

Sommermeyer V, Béneut C, Chaplais E, Serrentino ME, Borde V. 2013. Spp1, a member of the Set1 complex, promotes meiotic DSB formation in promoters by tethering histone $\mathrm{H} 3 \mathrm{~K} 4$ methylation sites to chromosome axes. Mol Cell 49: 43-54.

Sturtevant AH. 1915. Castle and Wright on crossing over in rats. Science 42: 342.

Sturtevant A. 1919. Contributions to the genetics of Drosophila melanogaster. III. Inherited linkage variations in the second chromosome. Carnegie Inst Wash Pub 278: 305-341.

Sun L, Wang J, Sang M, Jiang L, Zhao B, Cheng T, Zhang Q, Wu R. 2017. Landscaping crossover interference across a genome. Trends Plant Sci 22: 894-907.

Svetlanov A, Baudat F, Cohen PE, de Massy B. 2008. Distinct functions of MLH3 at recombination hot spots in the mouse. Genetics 178: 1937-1945.

Sym M, Roeder GS. 1994. Crossover interference is abolished in the absence of a synaptonemal complex protein. Cell 79: 283-292. 
Turner JM. 2007. Meiotic sex chromosome inactivation. Development 134: 1823-1831.

Varas J, Sánchez-Morán E, Copenhaver GP, Santos JL, Pradillo M. 2015. Analysis of the relationships between DNA doublestrand breaks, synaptonemal complex and crossovers using the Atfas1-4 mutant. PLoS Genet 11: e1005301.

Vargas E, McNally K, Friedman JA, Cortes DB, Wang DY, Korf IF, McNally FJ. 2017. Autosomal trisomy and triploidy are corrected during female meiosis in Caenorhabditis elegans. Genetics 207: 911-922.

Wagner CR, Kuervers L, Baillie DL, Yanowitz JL. 2010. xnd-1 regulates the global recombination landscape in Caenorhabditis elegans. Nature 467: 839-843.

Wang S, Zickler D, Kleckner N, Zhang L. 2015. Meiotic crossover patterns: Obligatory crossover, interference and homeostasis in a single process. Cell Cycle 14: 305-314.

Yelina NE, Choi K, Chelysheva L, Macaulay M, de Snoo B, Wijnker E, Miller N, Drouaud J, Grelon M, Copenhaver GP, et al. 2012. Epigenetic remodeling of meiotic crossover frequency in Arabidopsis thaliana DNA methyltransferase mutants. PLoS Genet 8: e1002844.
Yokoo R, Zawadzki KA, Nabeshima K, Drake M, Arur S, Villeneuve AM. 2012. COSA-1 reveals robust homeostasis and separable licensing and reinforcement steps governing meiotic crossovers. Cell 149: 75-87.

Youds JL, Mets DG, McIlwraith MJ, Martin JS, Ward JD NJ ON, Rose AM, West SC, Meyer BJ, Boulton SJ. 2010. RTEL-1 enforces meiotic crossover interference and homeostasis. Science 327: 1254-1258.

Zakharyevich K, Tang S, Ma Y, Hunter N. 2012. Delineation of joint molecule resolution pathways in meiosis identifies a crossover-specific resolvase. Cell 149: 334-347.

Zalevsky J, MacQueen AJ, Duffy JB, Kemphues KJ, Villeneuve AM. 1999. Crossing over during Caenorhabditis elegans meiosis requires a conserved MutS-based pathway that is partially dispensable in budding yeast. Genetics 153: 1271-1283.

Zetka MC, Rose AM. 1995. Mutant rec-1 eliminates the meiotic pattern of crossing over in Caenorhabditis elegans. Genetics 141: 1339-1349.

Zhang L, Wang S, Yin S, Hong S, Kim KP, Kleckner N. 2014. Topoisomerase II mediates meiotic crossover interference. $\mathrm{Na}$ ture 511: 551-556. 


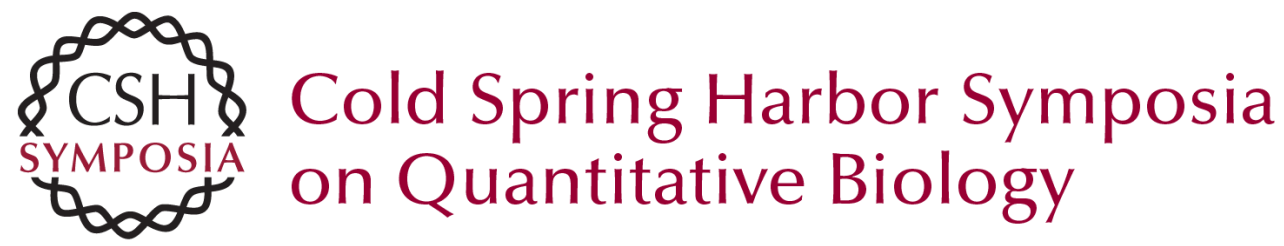

\section{Regulation of Crossover Frequency and Distribution during Meíotic Recombination}

Takamune T. Saito and Monica P. Colaiácovo

Cold Spring Harb Symp Quant Biol 2017 82: 223-234 originally published online December 8, 2017

Access the most recent version at doi:10.1101/sqb.2017.82.034132

References This article cites 106 articles, 41 of which can be accessed free at: http://symposium.cshlp.org/content/82/223.full.html\#ref-list-1

Creative This article is distributed under the terms of the

Commons http://creativecommons.org/licenses/by-nc/4.0/, which permits reuse and

License redistribution, except for commercial purposes, provided that the original author and source are credited.

Email Alerting

Receive free email alerts when new articles cite this article - sign up in

Service the box at the top right corner of the article or click here.

To subscribe to Cold Spring Harbor Symposia on Quantitative Biology go to: http://symposium.cshlp.org/subscriptions 